\title{
Simultaneous chronic rupture of quadriceps tendon and contra-lateral patellar tendon in a patient affected by tertiary hyperparatiroidism
}

\author{
Giuseppe Grecomoro • Lawrence Camarda • \\ Umberto Martorana
}

Received: 25 January 2007 / Accepted: 8 January 2008/Published online: 1 May 2008

(C) Springer-Verlag 2008

\begin{abstract}
Spontaneous ruptures of the extensor mechanism of the knee are very rare. They tend to increase considerably in patients with metabolic diseases such as chronic renal failure, hyperparathyroidism, diabetes, gout, and systemic lupus erythematosus. The reported case regards a 48-year-old man with chronic, spontaneous and simultaneous quadriceps, and contra-lateral patellar tendon rupture. The patient suffered from chronic renal failure and for the past year from tertiary hyperparathyroidism. Ruptured tendons were repaired and both knee were evaluated monthly for the next 12 months. Good functional recovery was achieved on both knees without relapse. This case emphasizes the importance of long-term high parathyroid hormone level in the etiology of tendons ruptures.
\end{abstract}

Keywords Patellar tendon rupture - Quadriceps tendon · Tertiary hyperparathyroidism

\section{Introduction}

Patellar tendon ruptures usually occur in athletic patients aged below 40 years, much more following sport injuries [1]. Its preferred location is the lower pole of the patella at

G. Grecomoro · L. Camarda · U. Martorana

Dipartimento delle Chirurgie Speciali, Unità Operativa di Ortopedia e Traumatologia, Università degli Studi di Palermo, Azienda Ospedaliera Universitaria Policlinico "P. Giaccone", Via del Vespro, 90127 Palermo, Italy

L. Camarda ( $₫)$

Sezione di Ortopedia e Traumatologia, Dipartimento delle Chirurgie Speciali, Università degli Studi di Palermo,

Via del Vespro, 90100 Palermo, Italy

e-mail: lawrencecamarda@hotmail.it the bone-tendon junction. Pre-existing patellar tendonitis and local corticosteroid injections are known to predispose to ruptures [2].

Quadriceps tendon rupture happens much more in correspondence with the osteotendinous junction usually following a fall or a trauma [3]. It occurs usually in subjects relatively old (sixth-seventh decades) with degenerative changes in the tendon.

Several systemic diseases predispose patients to spontaneous tendon rupture such as chronic renal failure in dialytic treatment, hyperparathyroidism, diabetes, rheumatoid arthritis, and systemic lupus erythematosus (SLE) [3-7].

Simultaneous rupture of quadriceps and contra-lateral patellar tendons represents an extremely rare association and just few cases were reported in medical literature $[7,8]$.

\section{Case report}

A 48-year-old man was admitted to our emergency room with acute onset of pain, massive effusions, and ecchymoses of both knees after a sudden fall happened about 1 month before. The patient referred to no direct or indirect trauma to the knees but just a subsiding of the left knee during walking and a secondary fall; on attempt to get up he realized that he was unable to move both knees actively.

The patient was affected by chronic renal failure in hemodialytic treatment for 2 years and for about a year he had suffered from tertiary hyperparathyroidism (THPT) with a constantly elevated parathyroid hormone (PTH) level $(>2,000 \mathrm{pg} / \mathrm{ml})$.

Inspection showed an alteration of the normal anatomic profile and swelling of both knees; the passive flexion and 
extension were painful bilaterally. On palpation, there was a gap in the proximal and distal regions of the left and right patellae, respectively (Fig. 1). Patellar floating was positive bilaterally.

The patient was unable to actively extend the knees from any flexed position and perform a straight leg raise in both knees. No other tendons, including the Achilles tendons, were found to be affected.

$\mathrm{X}$-ray examination of the knees was negative for bone lesions and revealed patella alta and patella baja on the right and left sides in the lateral projections, respectively (the Insall-Salvati index was 1.3 and 0.7, respectively) [9] (Fig. 2). Ultrasound examination of both knees showed a complete lesion of the distal and proximal insertions of the left quadriceps and right patella tendons, respectively. Bilateral MRI was performed and confirmed ultrasound results.

DEXA (dual energy X-ray absorptiometry) was also performed on the lumbar spine (L1-L4) and the hip which indicated osteoporosis (respectively, $T$ score was -4.20 and $-3.90 \mathrm{SD}$ ).

The hematochemical analysis revealed the following values: creatinine $12.85 \mathrm{mg} / \mathrm{dl}$ (normal range 0.50 $1.10 \mathrm{mg} / \mathrm{dl}$ ), urea $181 \mathrm{mg} / \mathrm{dl}(\mathrm{NR} 10-50 \mathrm{mg} / \mathrm{dl}$ ), alkaline phosphatase $170 \mathrm{UI} / \mathrm{l}$ (NR 50-190 UI/l), calcium 10,0 mg/ dl (NR 8.6-10.2 mg/dl), phosphorus $9.3 \mathrm{mg} / \mathrm{dl}$ (NR 2.7$4.5 \mathrm{mg} / \mathrm{dl}$ ), PTH $2,086 \mathrm{pg} / \mathrm{ml}$ (NR $10-60 \mathrm{pg} / \mathrm{ml}$ ), and $\beta, 2-$ microglobulin $1.8 \mathrm{mg} / \mathrm{l}$ (NR 0.6-3.8 mg/l).

Both knees were aspirated because of the hemarthrosis and were placed in above-knee braces until the operation. Bilateral reconstruction was performed 10 days after admission because of the clinical investigations.

Medial para-patellar incisions were made in both knees over the gaps of the right quadriceps and left patellar tendons.

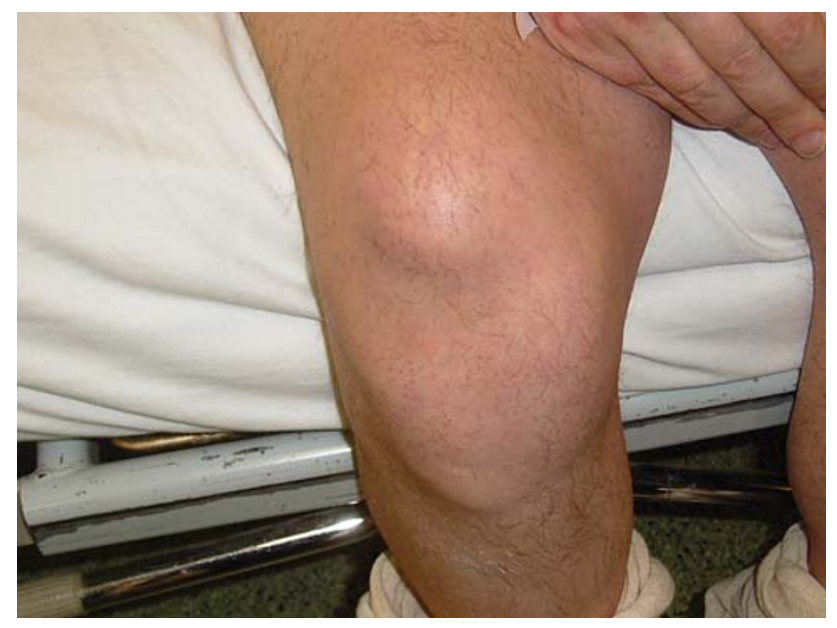

Fig. 1 Right knee, patella alta
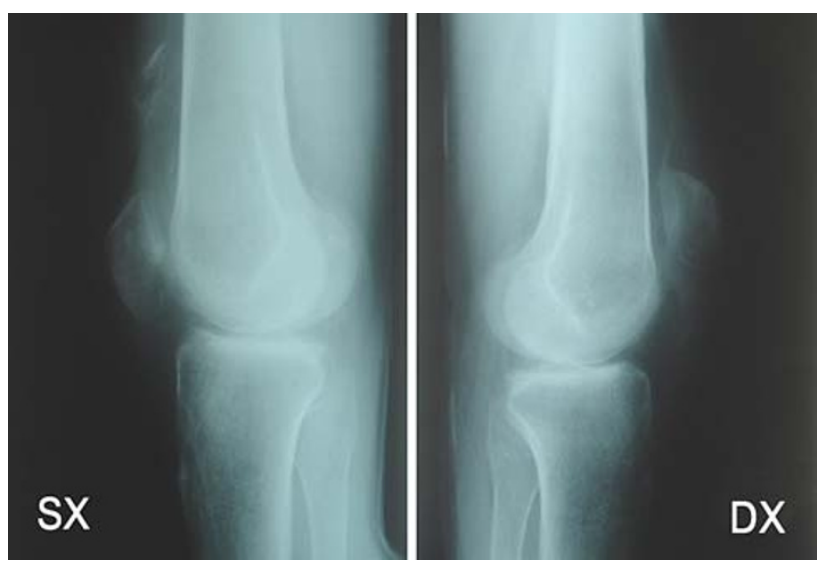

Fig. $2 \mathrm{Rx}$ of both knees shows patella alta on the right side and a patella baja on the left side

On the left side, the rupture of the quadriceps tendon was found and it was extended transversally to the lateral and medial retinaculum ligaments.

On the right side, we ascertained the complete rupture of the patellar tendon with a transverse rupture of the capsular ligaments in correspondence to the tendon injury, probably due to their stretch secondary to the chronic functional absence of the tendon.

The tendon's edges were rounded, sharpened, and not bleeding. The patellae appeared to be shining and were smooth to touch. We refreshed the tendon ends by bleeding their edges.

Following Krakow, on both knees non-absorbable sutures were passed through the tendon's edges, the retinacular ligaments and then through drill holes in the patellae [10]. On the left knee, Krakow's suture was preceded by a central lengthening of the quadriceps tendon according to Codivilla's Y/V technique in which a Vshaped incision is made in the quadriceps tendon allowing mobilization and closure of the gap with subsequent turndown of a tendon flap of the quadriceps tendon previously dissected on coronal plane [11]. On the right side, there was the possibility to restore the appropriate level of the patella. Therefore, we did not perform any surgical lengthening and release.

Post-operatively, both knees were immobilized in cylinder casts for 5 weeks followed by a brace for 60 days. Seven days after the operation, the patient started isometric quadriceps and straight leg raising exercises. Passive movement of the knees were begun 1 month post-operatively and at 5 weeks the patient started with partial weight bearing with crutches. Thereafter, rehabilitation exercises were intensified, active mobility was added to the rehabilitation program. Four months after the operation, the patient was able to walk on his own although one crutch was used as a precaution. 
The patient was clinically evaluated every week for 1 month and monthly for the next 7 months. The results achieved at 12 months were excellent in terms of pain and movement; the active full extension was possible on both knees and the bilateral active flexion was of $110^{\circ}$.

\section{Discussion}

Etiology of tendon ruptures is still not entirely understood. Tendon vascularization represents the most important factor that attends to the pathogenesis of those lesions. Following repetitive secondary microtears, the tendon vascularization tends to reduce because of thrombosis and infiltration of mononuclear cells. In this condition the tendons would become more susceptible to rupture during physical activity owing to the increased oxygen demanded by the tissue [12].

Inflammatory changes and elastosis can be observed at the site of rupture in patients, respectively, with SLE and chronic acidosis [13].

Renal failure with protracted hemodialysis can cause several complications that are linked to the dialytic process, such as amyloidosis (dialysis-related amyloidosis, DRA) [14]; in this particular type of amyloidosis there is an abnormal production of $\beta, 2$-microglobulin $(\beta 2-\mathrm{m})$. In healthy people $\beta 2-\mathrm{m}$ is metabolized by the kidneys, whereas in uremic patients, especially in those who underwent dialytic treatment with insufficiently permeable filters, it accumulates in blood with rates greater than 3040 times the normal values. In the DRA, the unexcretable plasma $\beta 2-\mathrm{m}$ tends to collect in particular areas such as joints, bones, and tendon structures [15]. This deposition causes a reduction of the tendon's elasticity and predisposes to rupture following minimal stress. Although we did not make an histochemical analysis of tendon tissue for the research of the $\beta 2-\mathrm{m}$, this is unlikely to be the cause of tendon rupture in our patient. In the DRA, in fact, the clinical manifestations almost never appear before 5 years of dialytic treatment; furthermore, our patient did not present high $\beta 2-\mathrm{m}$ blood values and did not show any features of the clinical triad characterizing musculoskeletal involvement in DRA (i.e. carpal tunnel syndrome, shoulder pain, and flexor tenosynovitis of the hands) [16].

Patient with chronic renal failure developed renal osteodystrophy and hyperparathyroidism characterized by general osteoporosis with subperiosteal bone resorption and secondary weakness of bone-tendon junction. This condition has been reported as the main cause of tendon ruptures [6].

Our patient was affected by severe osteoporosis and THPT, a type of hyperparathyroidism secondary to chronic renal failure not adequately compensated by therapy, in which PTH secretion is independent of the calcium serum concentration and autonomous parathyroid function has developed. This issue is confirmed by the very high levels of PTH during the previous year (even $>2,000 \mathrm{pg} / \mathrm{ml}$, NR 10-60). Our case was extremely rare because of the rapidity of the development of the hyperparathyroidism, about 12 months after the start of the dialytic treatment. Furthermore the intra-operative patellar aspect, the occurrence of rupture through the osteotendinous junction, and the relatively short duration of the hemodialytic treatment (for about 2 years) let us to hypothesize that the main cause of quadriceps and patellar tendons rupture in our patient is more likely to be the hyperparathyroidism and the longterm high PTH value. Our patient, 4 months after the tendons repair, underwent a subtotal parathyroidectomy with subsequent lowering of the PTH value.

Therefore we suggest careful clinical evaluation of the knee of all the patients with chronic renal failure which complain about knee pain, giving the opportunity for early surgical treatment.

Conflict of interest statement The authors declare that they have no conflict of interest related to the publication of this manuscript.

\section{References}

1. Anzel SH, Covey KW, Weiner AD et al (1959) Disruption of muscles and tendons: an analysis of 1014 cases. Surgery 45:406414

2. Wong MW, Tang YN, Fu SC, Lee KM, Chan KM (2004) Triamcinolone suppresses human tenocyte cellular activity and collagen synthesis. Clin Orthop Relat Res 421:277-281

3. Loehr J, Welsh RP (1983) Spontaneous rupture of the quadriceps tendon and patellar ligament during treatment for chronic renal failure. Can Med Assoc J 129:254-256

4. Bhole R, Johnson JC (1985) Bilateral simultaneous spontaneous rupture of quadriceps tendons in diabetic patient. South Med J $78: 486$

5. Morgan J, McCarty DJ (1974) Tendon ruptures in patients with systemic lups erythematosus treated with corticosteroids. Arthritis Rheum 17:1033-1066

6. Shiota E, Tsuchiya K, Yamaoka K et al (2002) Spontaneous mayor tendon ruptures in patients receiving long-term hemodialysis. Clin Orthop 394:236-242

7. Wener JA, Schein AJ (1974) Simultaneous bilateral rupture of the patellar tendon and quadriceps expansions in systemic lupus erythematosus: a case report. J Bone Joint Am 56:823-824

8. Rogers A, Rix S, Kulkarni R (2003) Simultaneous rupture of a patellar tendon and controlateral quadriceps tendon in a healty individual. Orthopedics 26:817-818

9. Insall J, Salvati E (1971) Patella position in the normal knee joint. Radiology 101:101-104

10. Krakow KA, Thomas SC, Jones LC (1986) A new stitch for ligament-tendon fixation. Brief note. J Bone Joint Surg Am 68:764-766

11. Scuderi C, Schrey EL (1950) Arch Surg 61:42

12. Davidsson L, Salo M (1969) Pathogenesis of subcutaneous tendon ruptures. Acta Chir Scand 135:209-212

13. Finlayson GR, Smith Jg Jr, Moore MJ (1964) Effect of chronic acidosis on connective tissue. JAMA 187:659-662 
14. Kurer MH, Baillod RA, Madgwick JC (1991) Muscoloskeletal manifestations of amyloidosis: a review of 83 patients on haemodialysis for at least 10 years. J bone Joint Surg Br 73:271-276

15. Masonis JL, Frick SL (2001) Bilateral quadriceps tendon ruptures as the initial presentation of amyloidosis. Orthopedics 10:995-996
16. Nangaku M, Miyata T, Kurokawa K (1999) Pathogenesis and management of dialysis-related amyloid bone disease. Am J Med Sci 317(6):410-415 